\title{
Resultados de voz y calidad de vida en pacientes con parálisis cordal unilateral en abducción tratados con tiroplastia de medialización
}

\author{
Voice and quality of life results in patients with unilateral vocal fold abduction paralysis \\ treated with medialization thyroplasty
}

Mario S. Dávalos-Fuentes* y Michelle E. de L. Marvin-Huergo

Servicio de Otorrinolaringología y Cirugía de Cabeza y Cuello, Instituto Nacional de Rehabilitación, Ciudad de México, México

\begin{abstract}
Resumen
Objetivo: Evaluar los resultados funcionales y el impacto en la calidad de vida de pacientes con parálisis cordal unilateral en abducción (PCUA) de etiología variable, posoperados de tiroplastia de medialización con técnica de Netterville en el Instituto Nacional de Rehabilitación. Método: En los pacientes candidatos a tiroplastia de medialización se documentaron el tiempo máximo fonatorio y cuestionarios de funcionalidad (VHI-30) y calidad de vida (VRQoL) prequirúrgicos y 3 a 6 meses posquirúrgicos. Se utilizaron pruebas de comparación de medias para analizar los resultados. Resultados: Se realizó tiroplastia de medialización en 27 pacientes con PCUA de 2013 a 2019. Todos tuvieron mejoría estadística y clínicamente significativa por el tiempo máximo fonatorio (media de 4.07 a 11.07 segundos), el VHI-30 (media de 81 a 29 puntos) y la VRQoL (media de 33 a 15 puntos). No se documentó ninguna complicación mayor. Conclusiones: La tiroplastia de medialización es un tratamiento seguro que mejora de manera significativa la calidad de vida y la funcionalidad de los pacientes con PCUA.
\end{abstract}

Palabras clave: Parálisis cordal. Tiroplastia de medialización. VHI. Tiempo máximo fonatorio. VRQoL.

\begin{abstract}
Objective: To evaluate functional and quality of life results in patients with unilateral vocal fold paralysis (UVFAP) of different etiology, who underwent medialization thyroplasty, using Netterville's technique. Method: In patients eligible for medialization thyroplasty, maximum phonatory time was measured, quality of life (VRQoL) and functionality (VHI-30) questionnaires were applied before surgery and posteriorly at 3 and 6 months after surgery. Means comparison tests were used to analyze the results. Results: Medialization thyroplasty was carried out in 27 patients presenting with UVFAP from 2013 to 2019. All had a statistically significant and clinically significant improvement. This was measure with maximum phonatory time (medium of 4.07 to 11.07 seconds), VHI-30 (medium of 81 to 29 points), and VRQoL (medium of 33 to 15 points). No mayor complications were documented. Conclusions: Medialization thyroplasty is a safe procedure that significantly improves functionality and quality of life in patients with UVFAP.
\end{abstract}

Key words: Vocal fold paralysis. Medialization thyroplasty. Maximum phonatory time. VHI. VRQoL.

\section{Correspondencia:}

*Mario S. Dávalos-Fuentes

Calzada México Xochimilco 289

Col. Arenal de Guadalupe, Alcaldía Tlalpan

C.P. 14389, Ciudad de México, México

E-mail: dr.mariodavalos@gmail.com
Cir Cir. 2021;89(4):443-448

Contents available at PubMed

www.cirugiaycirujanos.com

0009-7411/@ 2021 Academia Mexicana de Cirugía. Publicado por Permanyer. Este es un artículo open access bajo la licencia CC BY-NC-ND (http://creativecommons.org/licenses/by-nc-nd/4.0/). 


\section{Introducción}

La parálisis cordal unilateral en abducción (PCUA) representa un problema clínico debido a la discapacidad que provoca por su impacto en la voz, la deglución y la protección de la vía aérea'. Esto condiciona disfonía, aspiración, disnea y disfagia ${ }^{1-4}$, afectando de forma significativa la calidad de vida y la funcionalidad de los pacientes ${ }^{5}$.

La PCUA se debe a una lesión del nervio vago o de su ramo el nervio laríngeo recurrente, que por tener un trayecto más largo se presenta con mayor frecuencia en el lado izquierdo ${ }^{2,4}$. La causa más frecuente es la posquirúrgica, por procedimientos a lo largo de su trayecto, por lo que en primer lugar se reporta la cirugía de tiroides ${ }^{6}$, seguida por abordajes cervicales anteriores ${ }^{7}$, esofagectomía, cirugía cardiaca y mediastinoscopia'. Otras causas son trauma directo del nervio vago, intubación prolongada, neoplasias (tiroides, pulmón, aneurismas aórticos, condrosarcomas laríngeos), persistencia de conducto arterioso, radiación del cuello y causas sistémicas (varicela, herpes, enfermedad de Lyme, sífilis, sarcoidosis, lupus, amiloidosis, silicosis y afecciones neurológicas) ${ }^{1,8}$.

El tratamiento de la PCUA depende de la posibilidad de recuperación espontánea, la cual puede llegar a tardar de 6 a 12 meses principalmente en casos de paresia idiopática. Las opciones terapéuticas son observación con o sin terapia de voz, inyección laríngea (laringoplastia) con materiales temporales y cirugía del esqueleto laríngeo. La elección del tratamiento depende del paciente (profesionales de la voz, comorbilidad que eleve el riesgo quirúrgico), de la gravedad de los síntomas, de la etiología y del tiempo de evolución de la parálisis ${ }^{4,9}$.

Se ha descrito que los pacientes que reciben tratamiento temprano (< 6 meses) con inyección laríngea presentan un mejor pronóstico en competencia glótica y calidad de voz a largo plazo; sin embargo, no disminuye la necesidad de cirugía ${ }^{10,11}$. En aquellos pacientes con etiología posquirúrgica, la probabilidad de requerir tratamiento quirúrgico para la parálisis es mayor ${ }^{9}$.

Actualmente, la tiroplastia de medialización se considera el método de referencia para el tratamiento definitivo de la $\mathrm{PCUA}^{1,8}$. La cirugía fue descrita por Isshiki en 1974 como tiroplastia de tipo I para medialización de la cuerda vocal. La técnica consiste en la elaboración de una ventana en el borde anteroinferior del cartílago tiroides, permitiendo el acceso al espacio paraglótico con medición y colocación de una prótesis de silicón. El procedimiento se realiza con el paciente despierto, con anestesia local, y control endoscópico simultáneo con el fin de determinar el punto de medialización máxima requerido para una adecuada fonación ${ }^{12}$. Esta técnica es segura y eficaz, y logra un ajuste fino y graduado de la posición de la cuerda vocal para restaurar la función laríngea (protección, fonación y respiración) con un índice muy bajo de complicaciones (4-8\%) que incluyen edema, infección del sitio quirúrgico y extrusión $n^{13,14}$.

El índice de discapacidad vocal (VHI, Voice Handicap Index) es un cuestionario diseñado en 1997 por Jackobson que cuantifica el impacto subjetivo (percibido por el paciente) por un trastorno relacionado con la voz y que en 2007 fue validado en español (alfa de Cronbach: 0.93)

El cuestionario de calidad de vida relacionada con la voz (VRQoL, Voice-Related Quality of Life) fue diseñado en 1999 por Hogikyan y Sethuraman con el objetivo de medir el impacto en la calidad de vida en pacientes con trastornos de la voz (alfa de Cronbach: 0.91$)^{16}$.

El tiempo máximo fonatorio (TMF) es un examen clínico para valorar la disfonía descrito por Kent en $1987^{17}$. Para medirlo se solicita al paciente que realice una inspiración profunda y posteriormente sostenga una vocal; se realiza tres veces y se toma el promedio de tiempo, y un resultado $>8$ segundos se considera normal. El resultado depende de la competencia glótica y del volumen de aire disponible en los pulmones para la fonación ${ }^{17}$.

El objetivo de este estudio es reportar los resultados de voz utilizando el $\mathrm{TMF}^{3}$, la discapacidad medida por la escala $\mathrm{VHI}-30^{15}$ y la calidad de vida relacionada a la voz medida con el cuestionario VRQoL $L^{1}$ en pacientes con parálisis cordal unilateral en abducción tratados con tiroplastia de medialización.

\section{Método}

Estudio observacional, retrospectivo y longitudinal, aprobado por el comité de ética institucional.

Se realizó una revisión de expedientes de los pacientes con diagnóstico de PCUA en quienes se realizó una tiroplastia de medialización en el periodo de 2013 a 2019. Se incluyeron 27 pacientes, de los cuales reportamos los resultados funcionales y de calidad de vida. Se excluyeron los pacientes con diagnóstico de paresia cordal, parálisis cordal bilateral, aquellos no candidatos a tratamiento quirúrgico 
por comorbilidad importante y los que rechazaron el tratamiento quirúrgico.

\section{Técnica quirúrgica}

Todas las intervenciones fueron realizadas por el mismo cirujano (MSDF) con la técnica estandarizada descrita por Netterville, et al..$^{13}$ en 1993. Se utilizó una prótesis de Silastic de densidad 30 . El procedimiento se realizó bajo sedación y con anestesia local. Con el fin de asegurar una adecuada posición de la prótesis y evitar la medialización de la banda o el ventrículo, se corroboró la posición de la prótesis bajo control endoscópico con nasofaringolaringoscopia. El tamaño de la prótesis y el grado de medialización se determinaron por el cambio del TMF y la calidad de la voz obtenidos durante el procedimiento.

En estos pacientes se tomó el TMF y se aplicaron los siguientes cuestionarios validados al español:

- EI VHI-30, que toma en cuenta tres ámbitos: función vocal, capacidad física relacionada y efecto emocional que provoca la disfonía. Consiste en 30 ítems organizados en tres subescalas (funcional, física y emocional) con 10 ítems cada una. Cada inciso tiene una puntuación de 0 a 4 para medir la gravedad percibida, lo que da un puntaje máximo de 120 puntos; a mayor puntaje, mayor es la discapacidad del paciente. Existe una modificación con 10 ítems (VHI-10) que también se ha validado para medir la discapacidad ${ }^{15}$.

- EI VRQoL, que consta de 10 ítems que miden la funcionalidad física y aspectos socioemocionales. Cada ítem se califica de 1 a 5 por el paciente y el puntaje máximo es de 50 puntos; a mayor puntaje. mayor afección de la calidad de vida ${ }^{16,18 .}$

Se realizó laringoscopia indirecta con endoscopio de $70^{\circ}$, antes de la cirugía y posteriormente a los 1 , 3 y 6 meses después de la cirugía.

\section{Análisis estadístico}

Para el análisis estadístico de los resultados se utilizaron estadística descriptiva y la prueba t de Student con los programas Excel (Microsoft Corporation) y SPSS (IBM).

\section{Resultados}

Se estudiaron 27 pacientes con diagnóstico de PCUA, con una edad promedio de 45 años (rango:
Tabla 1. Características epidemiológicas

\begin{tabular}{llc}
\hline & & Rango (media) \\
\hline Edad, años & $13-68(45)$ \\
Tiempo de evolución, meses & $9-216(55)$ \\
\hline & Mujer & N (\%) \\
\hline Sexo & Hombre & $17(62)$ \\
Cuerda vocal paralizada & Izquierda & $10(38)$ \\
& Derecha & $18(66)$ \\
Etiología & Posquirúrgica & $17(62)$ \\
& Tumores & $4(14)$ \\
& Neurológica & $2(7)$ \\
& Idiopática & $4(14)$ \\
\hline
\end{tabular}

13-68 años), de los que 17 eran mujeres (62\%) y 10 eran hombres (38\%). Se encontró afección de la cuerda vocal izquierda en 18 (66\%) de los pacientes y de la derecha en 9 (33\%). La etiología más frecuente fue posquirúrgica en 17 (62\%), seguida de tumores cervicales en $4(14 \%)$, causas neurológicas en $2(7 \%)$ e idiopática en 4 (14\%) pacientes. El tiempo de evolución al tratamiento fue en promedio de 55 meses (rango: 9-216 meses), dependiendo del momento en que los pacientes fueron referidos a nuestro servicio. Estas características se resumen en la tabla 1.

Como se puede observar en la tabla 2, el TMF tuvo una mejoría de 7 segundos, con un resultado posquirúrgico medio de 11 segundos (Fig. 1). Un TMF $\geq 8$ segundos se considera normal y, por lo tanto, un éxito quirúrgico. Dos pacientes (7.4\%) presentaron un TMF < 8; sin embargo, mostraron una mejoría de 3 a 6 segundos y de 1 a 4 segundos, ambos pacientes con alteraciones de la ventilación pulmonar por secuelas de enfermedad vascular cerebral (EVC).

En cuanto a la funcionalidad, medida por el VHI-30, se reportó una disminución media de 52 puntos en el total (Fig. 2). Si consideramos cada subescala, encontramos una mejoría muy homogénea: en funcionalidad 17 puntos, física 16 puntos y emocional 18 puntos (Fig. 3). Una disminución de 18 puntos en total o de 8 puntos en cada subescala se considera clínicamente significativo. Solo $3(11.1 \%)$ pacientes tuvieron una mejoría menor de 18 puntos, uno de ellos por tener secuelas de EVC y los otros dos por presentar una calificación baja desde el preoperatorio. 
Cirugía y Cirujanos. 2021;89(4)

Tabla 2. Resultados antes y después de la cirugía

\begin{tabular}{|c|c|c|c|c|}
\hline Métrica (media $\pm \mathrm{DE})$ & Prequirúrgico & Posquirúrgico & Diferencia promedio & Prueba estadística y significancia \\
\hline VHI-30 total & $81 \pm 32.78$ & $29 \pm 16.41$ & 52 & $\begin{array}{c}t \text { de Student } \\
p=0.000\end{array}$ \\
\hline VHI funcional & $28.19 \pm 11.34$ & $11.88 \pm 6.7$ & 17.61 & $\begin{array}{l}\text { t de Student } \\
p=0.000\end{array}$ \\
\hline VHI físico & $28.38 \pm 10.87$ & $10.76 \pm 6.7$ & 16.30 & $\begin{array}{c}\mathrm{t} \text { de Student } \\
\mathrm{p}=0.000\end{array}$ \\
\hline VHI emocional & $24.42 \pm 12.24$ & $6.34 \pm 4.9$ & 18.07 & $\begin{array}{l}t \text { de Student } \\
p=0.00\end{array}$ \\
\hline VRQoL & $33.34 \pm 10.12$ & $15.53 \pm 5.36$ & 17.81 & $\begin{array}{c}\mathrm{t} \text { de Student } \\
\mathrm{p}=0.000\end{array}$ \\
\hline TMF & $4.07 \pm 1.5$ & $11.30 \pm 4.9$ & 7.23 & $\begin{array}{l}\text { Wilcoxon } \\
p=0.00\end{array}$ \\
\hline
\end{tabular}

DE: desviación estándar; TMF: tiempo máximo fonatorio; VHI: Voice Handicap Index; VRQoL: Voice-Related Quality of Life.

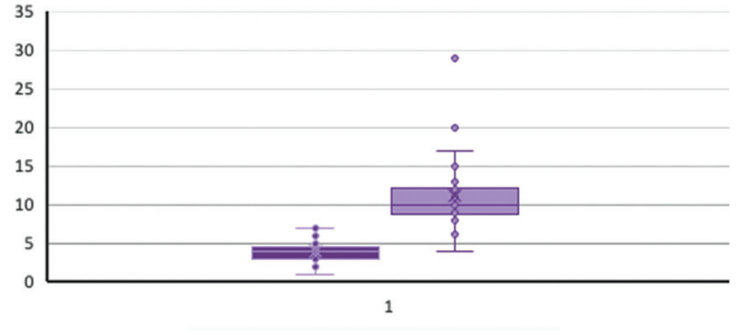

[ TMF prequirúrgico $\square$ TMF Posquirúrgico

Figura 1. Tiempo máximo fonatorio (TMF) antes y después de la cirugía.

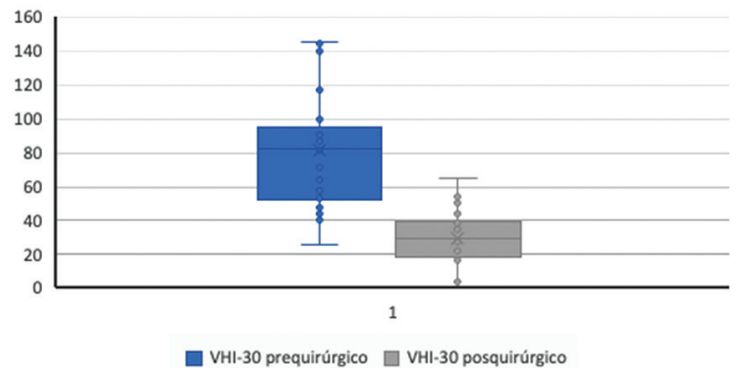

Figura 2. Resultados del Voice Handicap Index (VHI-30) antes y después de la cirugía.

En la calidad de vida medida por el VRQoL se obtuvo un cambio promedio de 17 puntos (Fig. 4). Un cambio de 8 puntos se considera clínicamente significativo. Con los tres parámetros medidos se puede hablar de éxito quirúrgico. Tres (11.1\%) pacientes no

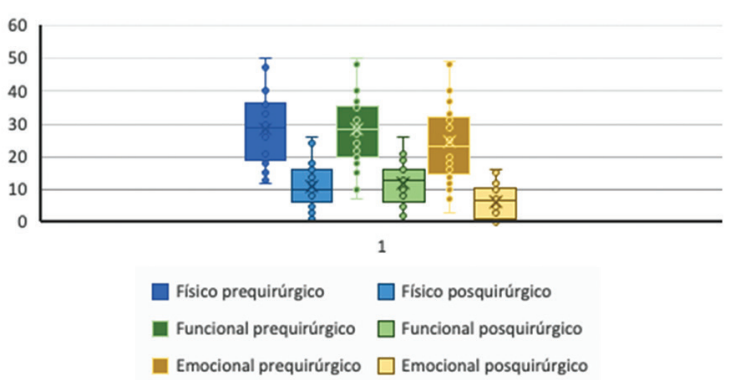

Figura 3. Resultados de las subescalas del Voice Handicap Index antes y después de la cirugía.

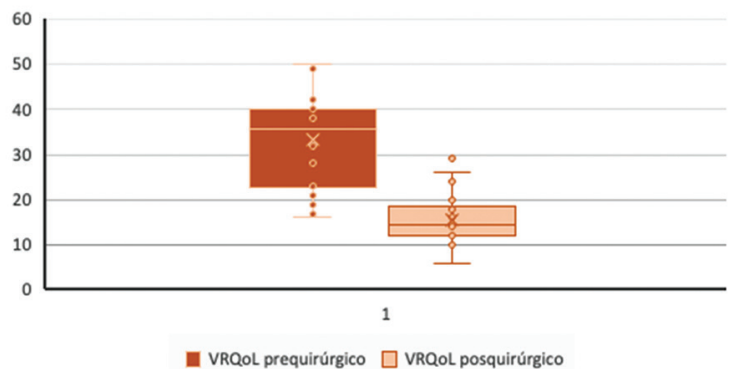

Figura 4. Resultados del cuestionario Voice-Related Quality of Life (VRQoL) antes y después de la cirugía.

tuvieron éxito en este parámetro, uno de ellos por secuelas de EVC, otro por calificación baja desde el preoperatorio y otra paciente con un cambio de 7 puntos, que contrasta con el VHI, en el que tuvo mejoría de 48 puntos. 
Tabla 3. Resultados del Voice Handicap Index (VHI) y del tiempo máximo fonatorio (TMF)

\begin{tabular}{|c|c|c|c|c|c|}
\hline Autor & Año y país & VHI precirugía & VHI poscirugía & TMF precirugía & TMF poscirugía \\
\hline Shen, et al. ${ }^{25}$ & 2013, USA (metaanálisis) & 72.2 & 34 & 6.16 & 12.4 \\
\hline Benninger, et al. ${ }^{24}$ & 2015, USA & 67 & 22 & 8.5 & 24.2 \\
\hline Aravena, et a ${ }^{23}$ & 2017, Chile & $33.2(\mathrm{VHI}-10)$ & $17.4(\mathrm{VHI}-10)$ & & \\
\hline Marvin, Dávalos & 2020, México & 81 & 29 & 4 & 11.3 \\
\hline
\end{tabular}

De los 27 pacientes, dos requirieron reintervención. La primera paciente se reintervino a los 2 meses de la cirugía inicial por medialización insuficiente, se realizó la misma técnica y se corrigió el punto máximo de medialización con buen resultado. El segundo paciente se reintervino debido a que 2 años después de la primera cirugía volvió a presentar datos de aspiración, se corroboró la adecuada posición de la cuerda vocal y se realizó una aducción de aritenoides, con lo que mejoró la sintomatología. Un paciente presentó extrusión de la prótesis, pero no requirió reintervención ya que aun después de la extrusión no volvió a presentar insuficiencia glótica. No se reportó ninguna complicación mayor.

\section{Discusión}

La parálisis cordal en abducción no compensada afecta de manera significativa la funcionalidad y la calidad de vida de los pacientes. Esto se debe a que presentan una alteración en la calidad de la voz y en la deglución, pero sobre todo una deficiencia en la protección de la vía aérea. Los episodios crónicos de aspiración son un factor de riesgo para neumonía de repetición ${ }^{19}$.

La parálisis cordal unilateral es una complicación frecuente en la cirugía de cuello, con mayor incidencia tras la cirugía de tiroides. En la actualidad existe la posibilidad de realizar cirugía de tiroides con neuromonitorización del nervio vago con el fin de disminuir el riesgo de lesionarlo; sin embargo, no se ha reportado una disminución estadísticamente significativa en la incidencia de lesiones del nervio laríngeo recurrente con el uso de neuromonitorización, a pesar de existir una tendencia a la baja y un mejor pronóstico en la rehabilitación de estos pacientes. Esto probablemente se deba a que conocer el estado del nervio en el transquirúrgico y el posquirúrgico inmediato permite tomar decisiones tempranas para determinar la mejor conducta terapéutica ${ }^{20,21}$.
En los pacientes con PCUA en quienes no se espera la recuperación neural, el tratamiento de elección es la tiroplastia de medialización por ser un procedimiento seguro, con un bajo índice de complicaciones y con buenos resultados inmediatos y a largo plazo. Nosotros utilizamos el TMF y el VHI-30 para medir de forma objetiva y subjetiva la discapacidad de los pacientes intervenidos, ya que son parámetros con un alto porcentaje de significancia, accesibles y sencillos de aplicar ${ }^{22}$. Además, utilizamos el VRQoL para medir el impacto sobre la calidad de vida de estos pacientes antes y después de la cirugía. Los resultados de nuestro centro son comparables con los reportados en la literatura (Tabla 3) ${ }^{23-25}$, probablemente porque todas las cirugías fueron realizadas por el mismo cirujano y con la misma técnica.

Entre los beneficios de la técnica de Netterville sobre la tiroplastia de medialización con colocación de implantes preformados se encuentran su bajo costo (con un solo bloque de silicón se pueden realizar hasta 10 cirugías) y la precisión de la medialización al tallar la prótesis según las necesidades específicas de cada paciente, lo cual también puede explicar el bajo índice de reintervenciones.

En cuanto a las limitaciones de nuestro estudio, mencionar que es una serie retrospectiva y por lo tanto no se pueden controlar las variables, y que contamos con una muestra pequeña, a pesar de ser un centro de referencia. Es difícil estandarizar el tiempo de la intervención, ya que la mayoría de nuestros pacientes son referidos desde otras instituciones.

\section{Conclusiones}

La PCUA no solo puede precipitar complicaciones como tos crónica, pérdida ponderal o neumonía por aspiración, sino que además se acompaña de una disminución de la calidad de vida por la discapacidad tanto física como emocional y social. 
Dado que la causa más frecuente de PCUA es posquirúrgica, el cirujano que maneja el nervio vago y sus ramos en cualquier región de su trayecto (base del cráneo, cuello y tórax) debe tomar conciencia de las implicaciones a corto y largo plazo de su lesión, y de las alternativas de tratamiento que existen y que mejoran de manera significativa la calidad de vida y la funcionalidad (TMF) de estos pacientes, entre las cuales la tiroplastia de medialización con técnica de Neterville ha demostrado ofrecer resultados rápidos en los pacientes en quienes no se espera la recuperación neural.

\section{Conflicto de intereses}

Los autores no cuentan con ningún conflicto de intereses.

\section{Responsabilidades éticas}

Protección de personas y animales. Los autores declaran que para esta investigación no se han realizado experimentos en seres humanos ni en animales.

Confidencialidad de los datos. Los autores declaran que han seguido los protocolos de su centro de trabajo sobre la publicación de datos de pacientes.

Derecho a la privacidad y consentimiento informado. Los autores declaran que en este artículo no aparecen datos de pacientes.

\section{Bibliografía}

1. Steglitz J, Warnick JL, Hoffman SA, Johnston W, Spring B. Evidence-based practice. Int Encycl Soc Behav Sci. 2015;45:332-8.

2. Walton $\mathrm{C}$, Conway $\mathrm{E}$, Blackshaw $\mathrm{H}$, Carding $\mathrm{P}$. Unilateral vocal fold paralysis: a systematic review of speech-language pathology management. J Voice. 2017:31:509.e7-22.

3. Uloza V, Pribuisiene R, Saferis V. Multidimensional assessment of functional outcomes of medialization thyroplasty. Eur Arch Oto-Rhino-Laryngology. 2005;262:616-21.

4. McLaughlin CW, Swendseid B, Courey MS, Schneider S, Gartner-Schmidt JL, Yung KC. Long-term outcomes in unilateral vocal fold paralysis patients. Laryngoscope. 2018;128:430-6.
5. Francis DO, Sherman AE, Hovis KL, Bonnet K, Schlundt D, Garrett CG, et al. Life experience of patients with unilateral vocal fold paralysis. JAMA Otolaryngol Head Neck Surg. 2018;144:433-9.

6. Jeannon JP, Orabi AA, Bruch GA, Abdalsalam HA, Simo R. Diagnosis of recurrent laryngeal nerve palsy after thyroidectomy: a systematic review. Int J Clin Pract. 2009;63:624-9.

7. Kriskovich MD, Apfelbaum RI, Haller JR. Vocal fold paralysis after anterior cervical spine surgery: incidence, mechanism, and prevention of injury. Laryngoscope. 2000;110:1467-73.

8. Bothe C, López M, Quer M, León X, García J, Lop J. Etiología y tratamiento de la parálisis laríngea: estudio retrospectivo de 108 pacientes. Acta Otorrinolaringol Esp. 2014:65:225-30.

9. Francis DO, Williamson K, Hovis K, Gelbard A, Merati AL, Penson DF, et al. Effect of injection augmentation on need for framework surgery in unilateral vocal fold paralysis. Laryngoscope. 2016;126:128-34.

10. Yung KC, Likhterov I, Courey MS. Effect of temporary vocal fold injection medialization on the rate of permanent medialization laryngoplasty in unilateral vocal fold paralysis patients. Laryngoscope. 2011;121:2191-4.

11. Costello D. Change to earlier surgical interventions: contemporary management of unilateral vocal fold paralysis. Curr Opin Otolaryngol Head Neck Surg. 2015;23:181-4.

12. Isshiki N. Progress in laryngeal framework surgery. Acta Otolaryngol. 2000;120:120-7.

13. Netterville JL, Stone RE, Civantos FJ, Luken ES, Ossoff RH. Silastic medialization and arytenoid adduction: the Vanderbilt experience. Ann Otol Rhinol Laryngol. 2014;102:413-24.

14. Laccourreye $\mathrm{O}$, Benkhatar $\mathrm{H}$, Meńard M. Lack of adverse events after medialization laryngoplasty with the Montgomery thyroplasty implant in patients with unilateral laryngeal nerve paralysis. Ann Otol Rhinol Laryngol. 2012;121:701-7.

15. Núñez-Batalla $F$, Corte-Santos $P$, Señaris-González $B$, Llorente-Pendás JL, Górriz-Gil C, Suárez-Nieto C. Adaptación y validación del índice de incapacidad vocal (VHI-30) y su versión abreviada (VHI-10) al español. Acta Otorrinolaringol Esp. 2007;58:386-92.

16. Hogikyan ND, Sethuraman G. Validation of an instrument to measure voice-related quality of life (V- RQOL). J Voice. 1999;13:557-69.

17. Pearl Solomon N, Garlitz SJ, Milbrath RL. Respiratory and laryngeal contributions to maximum phonation duration. J Voice. 2000;14:331-40.

18. Contreras F, González N, Vivero M, Guzmán M. Cross-cultural adaptation of the Chilean version of the Voice-Related Quality of Life (V-RQOL). Codas. 2019:31:1-6.

19. Tsai MS, Yang YH, Liu CY, Lin MH, Chang GH, Tsai YT, et al. Unilateral vocal fold paralysis and risk of pneumonia: a nationwide population-based cohort study. Otolaryngol Head Neck Surg. 2018;158:896-903.

20. Phelan E, Schneider R, Lorenz K, Dralle H, Kamani D, Potenza A, et al. Intraoperative nerve monitoring during thyroidectomy: evaluation of signal loss, prognostic value and surgical strategy. Laryngoscope. 2016;158:25-30.

21. Cirocchi R, Arezzo A, D'Andrea V, Abraha I, Popivanov GI, Avenia N, et al. Intraoperative neuromonitoring versus visual nerve identification for prevention of recurrent laryngeal nerve injury in adults undergoing thyroid surgery. Cochrane Database Syst Rev. 2019;(1):CD012483.

22. Desuter G, Dedry M, Schaar B, van Lith-Bijl J, van Benthem PP, Sjögren EV. Voice outcome indicators for unilateral vocal fold paralysis surgery: a review of the literature. Eur Arch Oto-Rhino-Laryngol. 2018;275:459-68.

23. Aravena $C$, Olavarría $L$, Zúñiga $P$, Sauvalle $C$, Cantillano $P$. Tiroplastía de medialización con Gore-Tex®: Experiencia de 8 años en Hospital Clínico de la Universidad de Chile. Rev Otorrinolaringol Cir Cabeza Cuello. 2017:77(1):9-15.

24. Benninger MS, Manzoor N, Ruda JM. Short- and long-term outcomes after silastic medicalization laryngoplasty: are arytenoid procedures needed? J Voice. 2015;29:236-40.

25. Shen T, Damrose EJ, Morzaria S. A meta-Analysis of voice outcome comparing calcium hydroxylapatite injection laryngoplasty to silicone thyroplasty. Otolaryngol Head Neck Surg. 2013;148:197-208. 